\title{
PERFILANDO LA PRÁCTICA DEL TELETRABAJO EN PANDEMIA: ESTUDIO GENERADO EN LOJA-ECUADOR
}

\section{PROFILING THE PRACTICE OF TELEWORKING IN PANDEMIC: STUDY GENERATED IN LOJA-ECUADOR}

\author{
Humberto Tapia Escalante* \\ Humberto Tapia Gómez ${ }^{*}$
}

\begin{abstract}
Resumen: Se realizó un estudio cuantitativo mediante la aplicación online de un cuestionario semiestructurado, para conocer en el contexto de la pandemia del Covid-19, el perfil de los trabajadores, sus actividades y ambientes de teletrabajo del Ecuador. El procesamiento de la información se efectuó utilizando el software SSPS y Excel. La muestra estudiada en su mayoría, tenía edades que fluctuaban entre los 30 y 60 años, poseía educación superior, y no había trabajado en modalidad de teletrabajo antes. Por otra parte, sobre el 60\% respondió convivir en sus hogares con entre 3 a 5 personas, que debe atender labores domésticas y trabaja más de 8 horas al día. Cabe mencionar que, más del 50\% manifestó haber aumentado su peso, no realiza actividad física y presenta dolores corporales. Se destacan como ventajas del teletrabajo la flexibilidad en los tiempos y menos costos y tiempo por desplazamientos. Se concluye que el teletrabajo no debe ser considerado solo como la interacción de un trabajador con un equipo informático, sino que debe ser desarrollado en el marco de una nueva forma de organización integral que contemple lo físico, cognitivo, emocional y la mitigación de afectaciones.
\end{abstract}

Palabras clave: Teletrabajo, trabajo en casa, teleworking.

\begin{abstract}
A quantitative study was carried out through the online application of a semi-structured questionnaire, to know in the context of the Covid-19 pandemic, the profile of workers, their activities and teleworking environments in Ecuador. The information processing was carried out using the SSPS and Excel software. The majority of the sample studied had ages ranging between 30 and 60 years, had a higher education, and had not worked telework before. On the other hand, over $60 \%$ responded to live in their homes with between 3 to 5 people, who must attend to housework and work more than 8 hours a day. It is worth mentioning that more than $50 \%$ stated that they had increased their weight, did not engage in physical activity, and presented body aches. The advantages of teleworking are the flexibility in time and less costs and time for travel. It is concluded that telework should not be considered only as the interaction of a worker with a computer equipment, but should be developed within the framework of a new form of integral organization that contemplates the physical, cognitive, emotional and the mitigation of affectations.
\end{abstract}

Keywords: Teleworking, telework at home, teleworking.

*Centro de Estudios del Trabajo. Loja, Ecuador. Correo electrónico: humbertotapia2010@yahoo.com. Orcid: https://orcid.org/oooo-0003-3740-7237. Autor de correspondencia.

${ }^{* *}$ Centro de Estudios del Trabajo. Loja, Ecuador. Correo electrónico: bebtog6@gmail.com. Orcid: https://orcid.org/oooo-0002-9788-6893 


\section{Introducción}

Etimológicamente, el término teletrabajo tiene como origen el griego tele de significado "lejos", y trabajo de raíz latina "tripaliāre" (de tripalı̆um), que hace referencia a un implemento de tortura hecho de tres palos, que alude más que al dolor al sufrimiento que ocasiona. Fue en la crisis energética de la década de los 70 en la que el físico Nilles (1976) denomina así a la actividad de trabajar desde casa u otro lugar, como opción a reducir el consumo de combustibles y contribuir a disminuir la contaminación ambiental: "si uno de cada siete trabajadores urbanos no tuviera que desplazarse a su lugar de trabajo, Estados Unidos no tendría la necesidad de importar petróleo” (pp. 4). En el año de 1982, funda JALA Internatinal Inc, constituyéndose en la primera consultora de teletrabajo del mundo, que asesoró a Gobiernos y empresas sobre esta práctica de trabajo.

En los inicios de esta original forma de trabajar, se la consideró como fuente de nuevas oportunidades para la ocupación de personas discapacitadas y otros grupos desfavorecidos, pues ayudó a la reducción de los costes laborales y al incremento de la productividad; incluso mejoró la calidad de vida de los teletrabajadores e incrementó su satisfacción laboral, además de disminuir el estrés (Angell, 2000). A la fecha, varios autores y estudios afirman que esta sublime visión o aspiración podría haber sido cumplida en alguna medida quizá por un pequeño grupo de trabajadores privilegiados, en algunas pocas empresas dispersas en el mundo (Barba, 2001; McLuhan et al., 1989; Toffler, 1980).

Más allá de esas consideraciones, en la actualidad se pueden contar con múltiples definiciones de lo que es el teletrabajo. Se citan algunas:

"El teletrabajo es una forma flexible de organización del trabajo, que consiste en el desempeño de la actividad profesional sin la presencia física del trabajador en la empresa durante una parte importante de su horario laboral. Engloba una amplia gama de actividades y puede realizarse a tiempo completo o parcial. La actividad profesional en el teletrabajo implica el uso permanente de algún medio de telecomunicación para el contacto entre el teletrabajador y la empresa" (Gray et al., 1995, pp. 73).

"Una forma de organizar el trabajo de manera que este se realiza con ayuda de las tecnologías de la información y las comunicaciones, en un lugar distinto y alejado de las oficinas centrales o de las instalaciones de producción” (Ley 1221 de 2008, artículo 2).

Ortiz-Chaparro (1996), en su libro El teletrabajo: Una nueva sociedad laboral en la era de la tecnología, dice: "teletrabajo es trabajo a distancia, utilizando las telecomunicaciones y por cuenta ajena".

Como elemento indispensable para el teletrabajo siendo este remoto, es que implica el uso de tecnologías de la información y la comunicación (TIC) (Gillespie \& Feng, 1994; Kerrin \& Hone, 2001; Wikström et al., 1997). La denominación de telework es más frecuente por algunos autores americanos y la mayoría de los europeos (Huws et al., 1990). 
Se ha contemplado dentro de la definición a una gran variedad de locaciones (telecentros, telecottages, oficinas satélite) y se enfatiza la distancia (Baruch, 2000; Lamond et al., 1998), entendiéndose como una ubicación espacial alejada del sitio físico que la organización destina habitualmente a sus empleados, o bien una ubicación alejada del sitio donde se entrega el resultado final del trabajo (Vilaseca, 2004). Se podría resumir que las definiciones giran en torno al lugar de trabajo, uso de tecnologías, el tiempo de dedicación y la relación laboral.

La Asociación Internacional de Teletrabajo (ITAC) señala que el teletrabajo es "una adaptación del trabajo en que los empleados trabajan en un sitio alternativo al menos 8 horas cada dos semanas y que facilita la reducción del tiempo y la distancia mediante la conexión con los empleados" (Vilaseca, 2004, pp. 16).

La Organización Internacional del Trabajo ([OIT], 2020) define al teletrabajo como "cualquier trabajo efectuado en un lugar lejos de las oficinas o de los talleres centrales, el trabajador no mantiene un contacto personal con sus colegas, pero puede comunicarse con ellos a través de las nuevas tecnologías". El alcance del convenio 177 sobre el trabajo a domicilio de 1996 de la OIT, incluye el "teletrabajo" cuando se lleva a cabo como un arreglo continuo o permanente, ya sea a tiempo completo o parcial.

Entre las definiciones se utilizan también términos como "telecommuting" y "teleworking", sin embargo, tiene acepciones particulares; el "telecommuting" hace referencia a trabajadores de la empresa o institución que laboran fuera de las instalaciones físicas, mientras que el "teleworking" hace referencia a quien trabaja para la empresa, fuera de ella como colaborador independiente (Departamento de Ergonomía de la Universidad de Concepción [UDEC] \& Sociedad Chilena de Ergonomía [SOCHERGO], 2020, pp.3).

Toffler (1980), destacado por sus aportes como escritor, sociólogo y futurista estadounidense; doctorado en letras, leyes y ciencia; reconocido por sus aportes a las discusiones sobre la revolución digital, la revolución de las comunicaciones y la singularidad tecnológica, predijo que el progreso en las tecnologías de telecomunicaciones, junto con el aumento de los costos asociados con los desplazamientos, marcaría el comienzo de la era de la "casa de campo electrónica", en la que el trabajo volvería al hogar, donde había estado durante gran parte de la civilización. Para el 2019, cuarenta años después de este anuncio, la proporción global de empleados que teletrabajaban de forma permanente era solo del $3 \%$ (OIT, 2021a, pp.111).

El escenario de pre-pandemia presentaba al teletrabajo en su mayor parte como ocasional y como mecanismo para extender las horas de trabajo (Noonan \& Glass, 2012). Según la OIT (2021b, pp. 124), en Australia el 43 por ciento de los trabajadores, que informó que laboraron en casa en 2017, afirmó que lo hacían para "ponerse al día con el trabajo". En los Estados Unidos, el 25 por ciento de los trabajadores asalariados trabajaron en casa al menos ocasionalmente en 2017-2018, pero solo el 2 por ciento lo hizo cinco días a la semana. En Japón, en 2016, una encuesta del Gobierno a 2.032 empresas con más de 100 empleados encontró que solo el $13,2 \%$ de las empresas había adoptado algún tipo de sistema de teletrabajo, y el trabajo móvil constituía dos tercios de los casos. Como resultado de una encuesta realizada por Japan Association for Chief Financial Officers ([JAHO], 2020, 6 de 
abril) antes del anuncio del estado de emergencia del 7 de abril de 2020, se encontró que el 31 por ciento de las empresas no podían adoptar el teletrabajo porque el papeleo aún no estaba digitalizado, las reglas y procedimientos internos necesarios para el teletrabajo no estaban listos (Akio, 2019).

Los antecedentes históricos en el mundo sobre la práctica de teletrabajo no son muy nutridos, hay áreas, tiempos, condiciones particulares que han permitido su aplicación; según la Fundación Europea para la Mejora de las Condiciones de Vida y de Trabajo (Eurofound) y OIT (2019) en la Unión Europea (UE), la incidencia del teletrabajo regular u ocasional (teletrabajo en el hogar y teletrabajo móvil combinados) variaba del 30 por ciento o más en Dinamarca, los Países Bajos y Suecia, y al 10 por ciento o menos en la República Checa, Grecia, Italia y Polonia. Según los estudios, hasta un 20 por ciento de la fuerza laboral de los Estados Unidos trabajaba regular $\mathrm{u}$ ocasionalmente desde su casa $\mathrm{u}$ otro lugar alternativo, un 16 por ciento en Japón y solo un 1,6 por ciento en Argentina.

Como resultado de las directivas de confinamiento decretadas por los Gobiernos, casi 4 de cada 10 empleados en Europa comenzaron a teletrabajar (Eurofound, 2020, 6 de mayo). El aumento más significativo del teletrabajo tuvo lugar en los países más afectados por el virus y en los que el teletrabajo estaba bien desarrollado antes de la pandemia. En Finlandia, cerca del 60 por ciento de los empleados pasaron a trabajar desde casa. En Luxemburgo, los Países Bajos, Bélgica y Dinamarca, más del 50 por ciento, en Irlanda, Austria, Italia y Suecia, alrededor del 40 por ciento de los empleados teletrabajaban. En esos países, menos trabajadores vieron una reducción en su tiempo de trabajo. En promedio, en Europa, el 24 por ciento de los empleados, que nunca habían trabajado desde casa, comenzó a teletrabajar, en comparación con el 56 por ciento de los empleados que lo habían hecho ocasionalmente.

Así como en otros continentes, el tiempo de pandemia ocasionó un despegar del teletrabajo en las más diversas áreas, motivado por realidades como la de los países de Latinoamericanos. La CEPAL reporta que se produjo un aumento de la tasa de desocupación de al menos 3,4 puntos porcentuales, hasta alcanzar una tasa del 11,5 por ciento, lo que equivale a más de 11,5 millones de nuevos desempleados (OIT, 2020, 26 de marzo).

Brasil, Costa Rica, Panamá, El Salvador y Chile ajustaron su legislación para permitir y fomentar el teletrabajo. Colombia tiene normado el teletrabajo en la Ley 1221 de 2008 y, en países como Paraguay y Ecuador, mediante decretos o leyes de emergencia regulan su aplicación. Según una encuesta realizada a 250 grandes empresas de la Argentina, el 93 por ciento de ellas había adoptado una política de teletrabajo como respuesta a la pandemia (Programa de las Naciones Unidas para el Desarrollo [PNUD], 2020, 30 de marzo).

En el contexto de estas realidades mundiales y latinoamericanas en tiempo de pandemia, el objetivo de este estudio es conocer el perfil de los trabajadores, actividades y ambientes de teletrabajo en el Ecuador y, particularmente, en el austro del país. 


\section{Materiales y métodos}

El estudio realizado fue descriptivo, transversal, cuantitativo y, de aplicación online, mediante encuestas con un cuestionario semiestructurado generado en Drive, enviado vía redes sociales, contactos y correos institucionales. Se obtuvieron respuestas de teletrabajadores de empresas e instituciones de la provincia y ciudad de Loja, Zamora Chinchipe, Quito, Imbabura, y Azuay. El grupo objetivo estaba constituido por trabajadores, empleados, directivos, hombres y mujeres de empresas públicas e instituciones privadas, que han laborado en teletrabajo no menos de 6 meses; la fecha de realización fue entre el 28 de noviembre al 6 de diciembre de 2020.

Se realizó una revisión detallada de las encuestas, y se incluyó solamente las que precisaban claramente su empleador y concordaban con los identificadores a los que fueron enviadas; en total el número de empresas y/o instituciones reflejadas en el estudio fueron 40 con 180 encuestas debidamente registradas. El análisis estadístico descriptivo se realizó con el programa SPSS estableciendo porcentualmente tendencias y la graficación mediante Excel.

\section{Resultados y discusión}

La población vinculada a las empresas reflejadas en el estudio, en un $80 \%$, están entre los 30 a los 60 años de edad, el 59\% es de sexo femenino, el 64\% está casado(a), el 64\% convive entre 3 a 5 personas en su domicilio, en un $65 \%$ con adultos.

Tabla 1. Porcentaje de personas por hogar según cantidad de habitantes.

\begin{tabular}{|c|c|c|c|}
\hline Número personas misma casa & $<\mathbf{3}$ & $\mathbf{3} \mathbf{A} 5$ & $>\mathbf{5}$ \\
\hline \% & 24 & 64 & 12 \\
\hline
\end{tabular}

Tabla 2. Porcentaje de personas que habitan el mismo hogar según rangos de edades.

\begin{tabular}{|c|c|c|c|}
\hline Edad habitan mismo hogar & Adultos & $\mathbf{1 1}$ a 18 años & $<$ 11 años \\
\hline \% & 65 & 20 & 15 \\
\hline
\end{tabular}

La superficie del hogar, en el $27 \%$, está entre los 101 a $140 \mathrm{~m}^{2}$; las actividades que impiden un buen desarrollo del teletrabajo en un $67 \%$ son la cocina y los niños.

Tabla 3. Porcentaje de acuerdo a la superficie del hogar.

\begin{tabular}{|c|c|c|c|c|c|}
\hline${\text { Superficie hogar } \mathbf{~ m}^{\mathbf{2}}}^{\mathbf{2}}$ & $<\mathbf{5 0}$ & $\mathbf{5 0}$ a 80 & $\mathbf{8 1}$ a 100 & $\mathbf{1 0 1}$ a 140 & $>\mathbf{1 4 0}$ \\
\hline \% & 13 & 13 & 17 & 27 & 30 \\
\hline
\end{tabular}

Tabla 4. Porcentaje de las actividades que interfieren con el teletrabajo.

\begin{tabular}{|c|c|}
\hline Actividades que interfieren con el teletrabajo & \% \\
\hline Cocina & 38 \\
\hline Cuidado niños & 29 \\
\hline Otras & 33 \\
\hline
\end{tabular}


El 95\% tiene estudios universitarios, su relación laboral es 52\% en instituciones públicas, el 53\% labora totalmente en teletrabajo, 65\% más de 8 horas al día, el $72 \%$ no recibió instrucciones sobre cómo ejecutar sus tareas y el $80 \%$ no tiene entrenamiento o capacitación en ergonomía.

Tabla 5. Caracterización sector y relación laboral.

\begin{tabular}{|c|c|}
\hline Sector laboral & \% \\
\hline Educativo & 42 \\
\hline Manufactura & 16 \\
\hline Salud & 8 \\
\hline Comercio & 5 \\
\hline Transporte & 3 \\
\hline Servicios públicos & 3 \\
\hline Otros & 23 \\
\hline Relación laboral & $\mathbf{\%}$ \\
\hline Contrato temporal & 55 \\
\hline Contrato definitivo & 45 \\
\hline Institución pública & 52 \\
\hline Empresa privada & 48 \\
\hline
\end{tabular}

Tabla 6. Caracterización experiencia previa y capacitación y/o conocimiento en ergonomía.

\begin{tabular}{|c|c|c|}
\hline Experiencia y formación & Sí (\%) & No (\%) \\
\hline Antes de pandemia teletrabajo & 12 & 88 \\
\hline Recibió capacitación teletrabajo & 28 & 72 \\
\hline Conoce de ergonomía & 20 & 80 \\
\hline
\end{tabular}

Tabla 7. Porcentaje de dedicación al teletrabajo por día.

\begin{tabular}{|c|c|c|}
\hline Dedicación laboral al teletrabajo & Total & Parcial \\
\hline \% & 53 & 47 \\
\hline
\end{tabular}

Tabla 8. Horas dedicadas al teletrabajo por día.

\begin{tabular}{|c|c|c|c|}
\hline Horas teletrabajo & $<\mathbf{4}$ & $\mathbf{4 a ~ 8}$ & $>\mathbf{8}$ \\
\hline $\mathbf{\%}$ & 15 & 20 & 65 \\
\hline
\end{tabular}

Por otra parte, la tecnología utilizada para teletrabajo, en $41 \%$, es un PC fijo y $34 \%$ portátil. Como elementos de apoyo cuentan el $76 \%$ con escritorio, $32 \%$ silla tipo oficina, $6 \%$ reposapiés y $22 \%$ con elevador de monitor. 
Figura 1. Tecnologías y apoyos disponibles para la gestión del teletrabajo en el hogar.

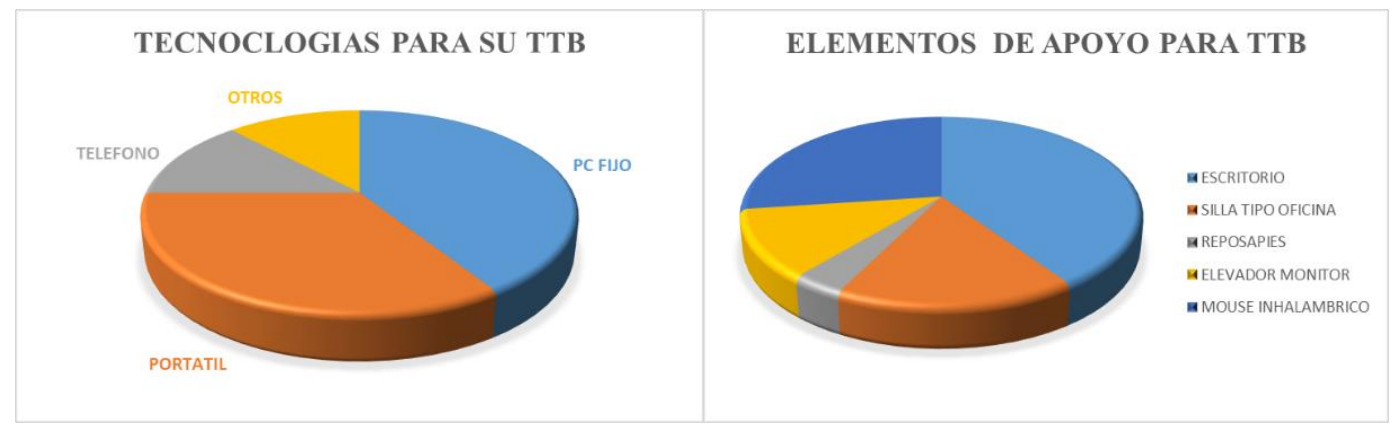

A un 55\% y 25\% no le brinda confort el ruido y la iluminación respectivamente, al 23\% le preocupa que le cambien sus labores contra su voluntad, y al $41 \%$ le preocupa perder su trabajo o sus clientes.

Figura 2. Caracterización teletrabajo y disconfort ambiental.

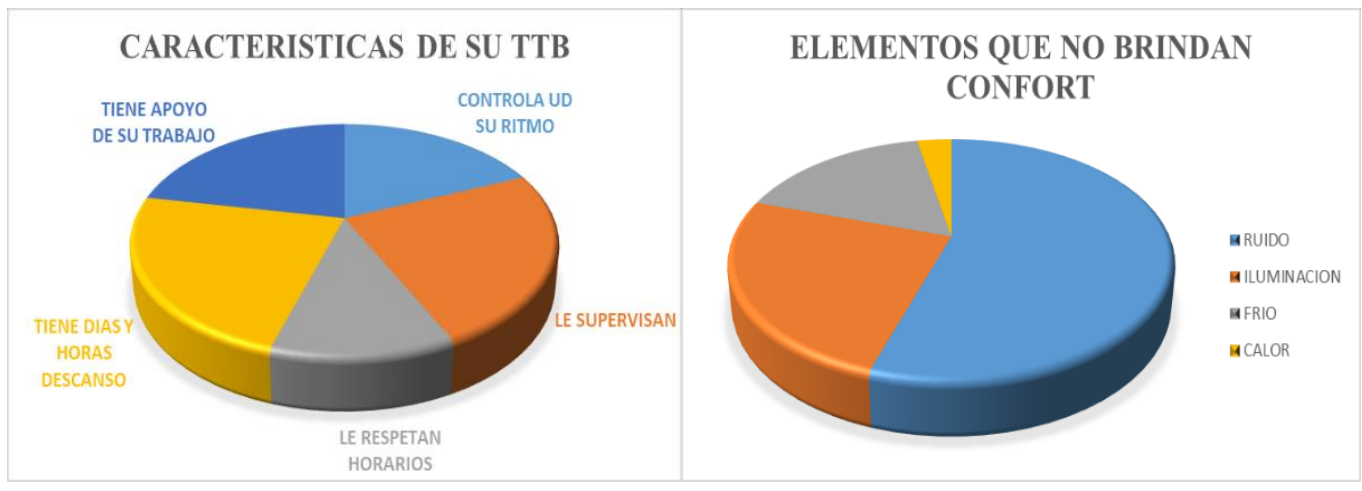

Entre el 49\% y 66\% no percibe comodidad en ningún segmento del cuerpo al realizar teletrabajo y presentan molestias entre el $40 \%$ y $70 \%$.

Figura 3. Segmentos afectados y dolores físicos presentados durante el teletrabajo.

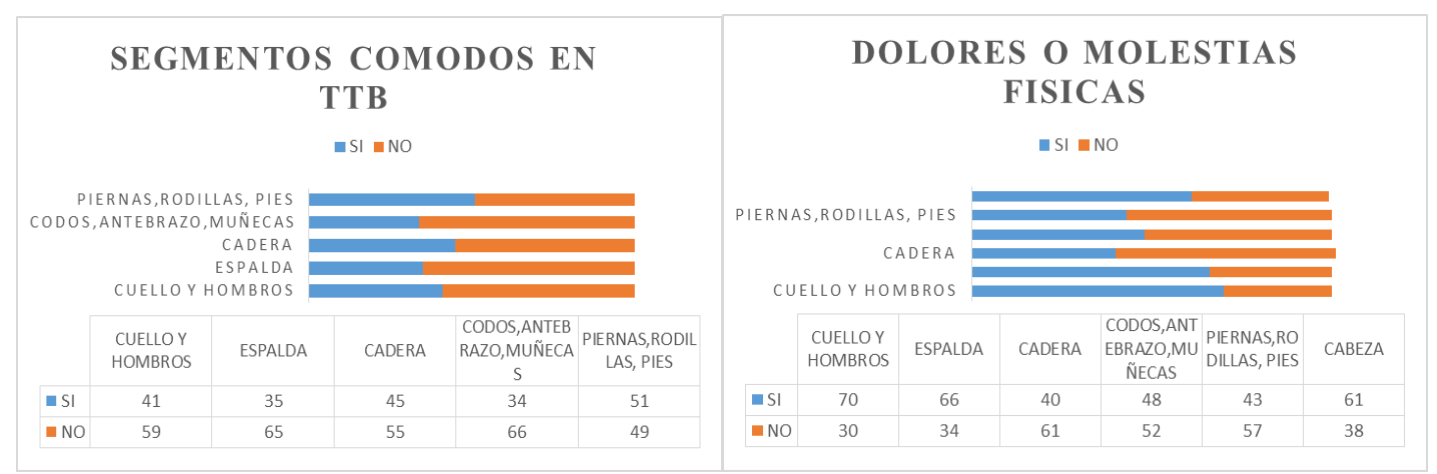

Más del 50\% consume más alimento y ha incrementado su peso. Entre el 40 y $90 \%$ no realiza actividad física. 
Figura 4. Impacto del teletrabajo sobre la alimentación y actividad física.

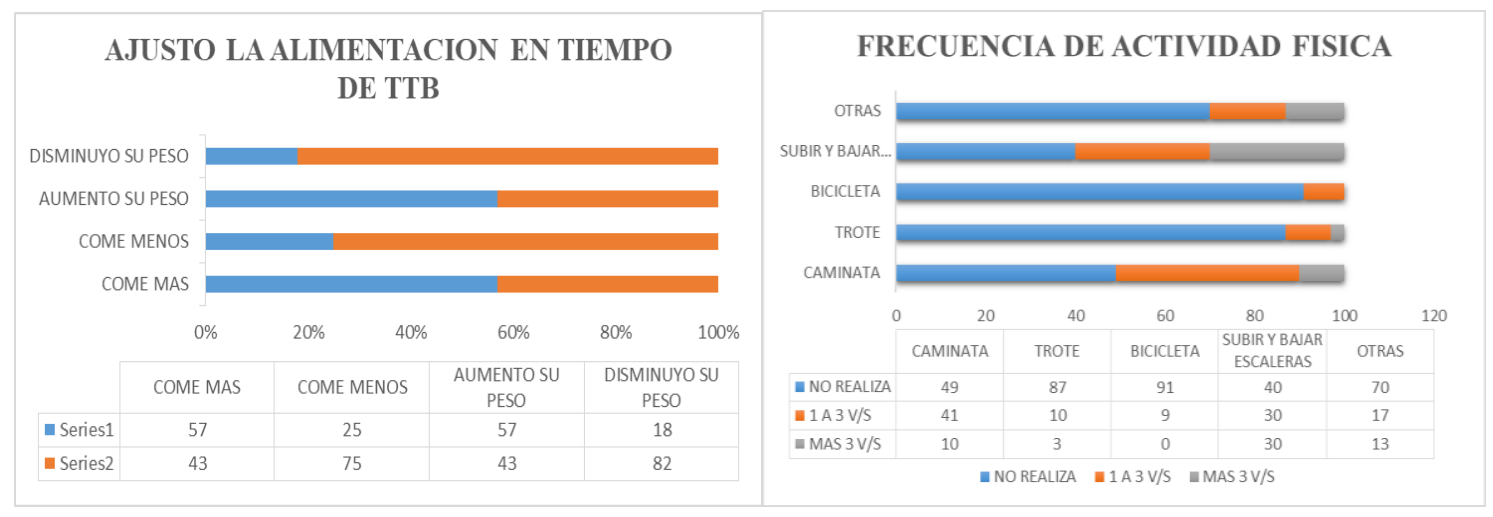

El 28\% encuentra como ventajas la flexibilidad en los tiempos de teletrabajo, y el $47 \%$ menor tiempo y costos en desplazamientos. Por otra parte, entre un $20 \%$ y $60 \%$, se ha(n) implementado alguna(s) medida(s) de mitigación en su trabajo.

\section{Discusión}

El teletrabajo aplicado como modalidad emergente frente a la pandemia, sin una cultura organizacional que lo haya tenido diseñado e implementado, aunque sea en pequeña escala, en la mayor parte de las organizaciones se convirtió en un conjunto de acciones laborales en un ambiente no acondicionado físicamente, ni dotado tecnológicamente para asumir este nuevo rol, generando nuevas realidades laborales como las que se reflejan en el estudio con un $65 \%$ de los encuestados que dicen trabajar más de 8 horas diarias.

Esto coincide con lo reportado por el informe Eurofound y OIT (2019), donde se menciona que el teletrabajo, en general, puede dar lugar a un horario de trabajo más prolongado y a un aumento de la carga de trabajo durante las noches y los fines de semana. Alude también a la interferencia entre el trabajo y la vida del hogar, lo que provoca efectos adversos en el bienestar y los niveles de estrés de los teletrabajadores; el estudio determina que más del 70\% de la población encuestada tiene que realizar actividades domésticas tipo cocina y atender a los niños a la par de las actividades laborales.

Como lo destacan Robertson y Mosier (2020), "las características físicas y ambientales de la oficina en el hogar y los hábitos de trabajo conexos son fundamentales para la capacidad de los trabajadores para realizar sus tareas a distancia y con eficacia”. En el estudio, en cuanto a la previsión en temas de seguridad y salud, el 25\% dice contar con algún nivel de protección, entre un 10 al 50\% menciona tener elementos para desarrollar teletrabajo en casa. Esto conlleva a que presenten afectaciones en su salud tipo dolores musculoesqueléticos, entre el 40 al 60\% de la población estudiada.

En cuanto al conocimiento y medios ergonómicos para el teletrabajo del estudio "entorno ergonómico del teletrabajo en situación de pandemia" realizado mediante encuesta en línea desarrollada por el Departamento de Ergonomía de la UDEC y la SOCHERGO (2020), se destaca que un 34,6\% de las personas encuestadas dice tener entrenamiento y/o capacitación en ergonomía y elementos de apoyo para realizar el teletrabajo, y entre un 20 al 
60\% cuenta con alguno o algunos de ellos tipo PC fijo, sillón, escritorio, reposapiés, etc., resultados comparativamente muy similares a los del presente estudio. Sin embargo, de todo lo antes citado, Cartmill (2020) reporta que en las encuestas realizadas con personas que trabajaron desde su casa durante la pandemia, se revela que también hay experiencias positivas con el trabajo desde el hogar, lo que en la presente investigación se refleja en el componente de mejoras en las relaciones familiares en más del 40\% de la población.

Lo recomendable sería ampliar las investigaciones a diferentes tipos de poblaciones trabajadoras, para abarcar las más diversas realidades, su impacto actual como posterior a la pandemia.

\section{Conclusiones}

Se concluye que el teletrabajo no debe ser considerado solo como la interacción de un trabajador con un equipo informático, sino que debe ser desarrollado en el marco de una nueva forma de organización integral de trabajo que contemple lo físico, cognitivo, emocional y mitigación de afectaciones, en consideración que esta modalidad de trabajo tendrá que convivir atemporalmente con la convencional. 


\section{Referencias}

Akio, S. (2019). Telework and its effects in Japan. En Jon C. Messenger (ed.), Telework in the 21st century: An evolutionary perspective (pp. 76-127). Edward Elgar Publishing and ILO. https://www.ilo.org/global/publications/books/WCMS 723395/lang--en/index.htm

Angell, I. (2000). The new barbarian manifesto. How to survive the information age. Kogan Page.

Barba, L. (2001). El teletrabajo y los profesionales de la información. El profesional de la información, 10(4), 4-13. http://eprints.rclis.org/15631/

Baruch, Y. (2000). Teleworking: Benefits and pitfalls as perceived by professionals and managers. New Technology, Work and Employment, 15(1), 34-49. https://doi.org/10.1111/1468$005 \mathrm{X} .00063$

Cartmill, C. (2020, 28 de mayo). New survey shows $87 \%$ of staff wish to work from home in post lockdown world. News Letter. https://www.newsletter.co.uk/business/new-survey-shows87-staff-wish-work-home-post-lockdown-world-2864590

Congreso de la República de Colombia (2008, 16 de julio). Ley 1221. Por la cual se establecen normas para promover y regular el teletrabajo y se dictan otras disposiciones. Diario Oficial 47.052. http://www.secretariasenado.gov.co/senado/basedoc/ley 1221 2008.html

Departamento de Ergonomía de la Universidad de Concepción \& Sociedad Chilena de Ergonomía (2020). Entorno ergonómico del teletrabajo en situación de pandemia. https://www.sochergo.cl/wp-content/uploads/2020/o8/ERGONOMIA-Y-TELETRABAJOUDEC-SOCHERGO-2020.pdf

Fundación Europea para la Mejora de las Condiciones de Vida y de Trabajo (2020, 6 de mayo). Vida, trabajo $y \quad$ Covid-19. Primeras conclusiones. https://www.eurofound.europa.eu/es/publications/report/2020/living-working-and-covid19-first-findings-april-2020

Fundación Europea para la Mejora de las Condiciones de Vida y de Trabajo \& Organización Internacional del Trabajo (2019). Trabajar en cualquier momento y en cualquier lugar: Consecuencias en el ámbito laboral. Oficina Internacional del Trabajo. https://www.ilo.org/wcmsp5/groups/public/---ed protect/---protrav/--travail/documents/publication/wcms 712531.pdf

Gillespie, A. \& Feng, L. (1994). Teleworking, work organization and the workplace. En Robin Mansell (ed.), The management of information and communication technologies: Emerging patterns of control (pp. 261-272). Aslib.

Gray, M., Hodson, N., Gordon, G., \& otros (1995). El teletrabajo. Telecomunicaciones, ECTF y Fundación Universidad Empresa.

Huws, U., Korte, W. B. \& Robinson, S. (1990). Telework: Towards the elusive office. John Wiley. Japan Association for Chief Financial Officers (2020, 6 de abril). Survey on the impact of Covid19 on financial operations. https://www.cfo.jp/news/13196/

Kerrin, M. \& Hone, K. (2001). Job seekers' perceptions of teleworking: A cognitive mapping approach. New Technology, Work and Employment, 16(2), 130-143. https://doi.org/10.1111/1468-005X.00082

Lamond, D., Standen, P., \& Daniels, K. (1998). Contexts, cultures and forms of teleworking. En Gerard Griffin (ed.), Management theory and practice: Moving to a new era (pp. 145-157). Macmillan.

McLuhan, M., \& Powers, B. R. (1989). The global village: Transformations in world life and media in the 21st century. Oxford University Press. 
Nilles, J. M. (1976). The telecommunications-transportation tradeoff. Options for tomorrow (pp. 4). John Wiley \& Sons.

Noonan, M. C. \& Glass, J. L. (2012). The hard truth about telecommuting. Monthly Labor Review 135(6), 38-45. $\quad$ https://www.bls.gov/opub/mlr/2012/article/hard-truth-abouttelecommuting.htm

Organización Internacional del Trabajo (1996, 20 de junio). C177. Convenio sobre el trabajo a domicilio.

NORMLEX https://www.ilo.org/dyn/normlex/es/f?p=NORMLEXPUB:12100:0::NO::P12100 INSTRU MENT_ID:312322

Organización Internacional del Trabajo (2020). Observatorio de la OIT: El COVID-19 y el mundo del trabajo. $\quad$ https://www.ilo.org/wcmsp5/groups/public/---dgreports/--dcomm/documents/briefingnote/wcms 740981.pdf

Organización Internacional del Trabajo (2020, 26 de marzo). Claves para un teletrabajo eficaz durante la pandemia del COVID-19. https://www.ilo.org/global/about-theilo/newsroom/news/WCMS 740038/lang--es/index.htm

Organización Internacional del Trabajo (2021a). Working from home. From invisibility to decent work (pp. 111). Oficina Internacional del Trabajo. https://www.ilo.org/global/publications/books/forthcomingpublications/WCMS 765806/lang--en/index.htm

Organización Internacional del Trabajo (2021b). Working from home. From invisibility to decent work (pp. 124). Oficina Internacional del Trabajo. https://www.ilo.org/global/publications/books/forthcomingpublications/WCMS 765806/lang--en/index.htm

Ortiz-Chaparro, F. (1996). El teletrabajo. Una nueva sociedad laboral en la era de la tecnología. Mc Graw Hill.

Programa de las Naciones Unidas para el Desarrollo (2020, 30 de marzo). Encuesta: Respuestas de las empresas argentinas ante el impacto de la cuarentena general en sus operaciones. https://www.ar.undp.org/content/argentina/es/home/library/environment energy/PGEnc uestaCOVID19.html

Robertson, M. M., \& Mosier, K. (2020). Work from home: Human factors/ergonomics considerations for teleworking. Organización Internacional del Trabajo. https://www.ilo.org/wcmsp5/groups/public/---ed_protect/---protrav/--safework/documents/publication/wcms 742061.pdf

Toffler, A. (1980). The third wave. Morrow.

Vilaseca, J. (2004). El teletreball a Catalunya: Conceptes, tipologies, mètriques i polítiques. Consell de Treball, Econòmic i Social de Catalunya. https://www.uoc.edu/uocpapers/dt/esp/vilaseca.html

Wikström, T., Lindén, K. P. \& Michelson, W (1997). Hub of events or splendid isolation. The home as a context for teleworking. Lund University School of Architecture. 